\title{
ON A PROPOSITION OF VON NEUMANN
}

\author{
By Masahiro Nakamura and Hisaharu Umegaki
}

1. J. von Neumann wrote, without proof, the following proposition in his monumental paper [3: footnote 10, p. 123]: "We could show that the operation $x \rightarrow x^{|p| q \mid \cdot \ldots}$ depends only on $x$ and $A$ (and not on the sequence $\left.p, q, \cdots\right)$ ", where $A$ is an abelian $W^{*}$-subalgebra of a semi-finite factor $M$ acting on a separable Hilbert space $H$, and where $p, q, \cdots$ is a sequence of projections in $A$ generating the subalgebra $A$.

In this note, we shall give a proof of the above mentioned proposition as a consequence of a result due to one of the authors $[5 ; \S 4]$. Furthermore, we wish to give a characterization of a maximal abelian subalgebra in a semi-finite $W^{*}$-algebra by means of conditional expectation.

2. Let $M$ be a semi-finite $W^{*}$-algebra with regular gage $\mu$. For any $W^{*-}$ subalgebra $A$ of $M$ there exists a non-negative linear operation $x \rightarrow x^{8}$ (with $\left\|x^{e}\right\| \leq\|x\|$ from $M$ into $A$ satisfying that

$$
\begin{array}{llll}
\text { (i ) } x^{e}=I^{e} x & \text { for } & x \in A, & \text { (ii) } x^{* e} x^{\beta} \leq\left(x^{*} x\right)^{e} \quad \text { and } x^{e}=x^{e e}, \\
\text { (iii) } x_{\alpha}^{e} \uparrow x^{e} \quad \text { if } & x_{\alpha} \uparrow x, & \text { (iv) }\left(x^{e} y\right)^{e}=\left(x y^{e}\right)^{e}=x^{e} y^{e} ;
\end{array}
$$

cf. [1; Théorème 8]. When it satisfies $I^{e}=I$, we have called it to be normal expectation; cf. [2]. Further, the above operation $x \rightarrow x^{e}\left(\right.$ not always $\left.I^{e}=I\right)$ satisfies that

$$
\text { (v) } \mu\left(x^{e} y\right)=\mu\left(x y^{e}\right) \text { for every } x \in M \text { and } \mu \text {-integrable } y \in M \text {; }
$$

cf. [1]. Conversely if a non-negative linear operation $x \rightarrow x^{\varepsilon}$ (with $I^{\varepsilon} \leq I$ ) from $M$ into $A$ satisfies (i), (ii) and (v), then it also satisfies (iii), (iv) and $x^{\varepsilon}$ $=x^{e}$ for every $x \in M$, cf. [5; Theorem 1], i. e. the $x \rightarrow x^{\beta}$ is uniquely determined by $A$ and $\mu$. We shall call the operation $x \rightarrow x^{e}$ to be conditional expectation (of $x$ ) conditioned by $A$.

Let $p, q, \cdots, r$ be arbitary finite set of projections in $M$. According to von Neumann [3], we define the operations $x \rightarrow x^{\mid p}, x^{|p| q}$ from $M$ into itself such as $x^{\mid p}=p x p+(1-p) x(1-p), x^{|p| q}=\left(x^{\mid p}\right)^{\mid q}$, and by successive application $x^{|p| q|\cdots| r}$ is defined which are normal expectations. Furthemore, if $p, q, \cdots, r, \cdots$ is a sequence of mutually commutative projections in $M$, then it is possible to introduce an operation $x \rightarrow x^{i p|q| \cdots}$ for $\mu$-integrable operators $x \in M$, with respect to the metric convergence (i.e. $L^{2}$-mean convergence)

$$
x^{|p| q \mid \cdots}=\lim _{r} x^{|p| q|\cdots| r} ;
$$

Received September 27, 1956; in revised form October 20, 1956. 
cf. [2; Lemma 2.1.6] and [5; Theorem 5]. Let $C_{p}$ denote the associate subalgebra of the operation $x \rightarrow x^{\mid p}$, in the sense of Nakamuru-Turumaru [2], which is the set of all elements $x \in M$ with $x^{\prime p}=x$, then $C_{p}$ is a $W^{*}$ subalgebra of $M$ consisting of all elements which commute with $p$. It is known [5; Theorem 4] that the operation $x \rightarrow x^{|p| q|\ldots| r}$ coincides with the conditional expectation conditioned by $C_{p} \cap C_{p} \cap \cdots \cap C_{r}$, and that each element of $C_{p} \cap C_{q} \cap \cdots \cap C_{r}$ commutes with every $p, q, \cdots, r$ (it is to be noted that the process corresponds to the process of taking the diagonal block of finite matrix; cf.[3; Introduction]). Furthermore, we have

THEOREM 1. If $p, q, \cdots$ is a sequence of mutually commutative projections in $M$, then the operation $x \rightarrow x^{|p| q \mid \cdot \cdot}$ defined on the algebra $M_{\mu}$ consisting of $\mu-$ integrable $x \in M$ is uniquely extended to the conditional expectation $x \rightarrow x^{e}$ on $M$ conditioned by

$$
C=C_{p} \cap C_{q} \cap \cdots
$$

and $x \rightarrow x^{e}$ is independent of the choice of the regular gage $\mu$, i.e. (v) holds for any regular gage of $A$, and $C$ is the set of all elements in $M$ commuting with $p, q, \cdots$.

It is sufficient to prove the conditional expection $x \rightarrow x^{e}$ being independent of the choice of the gage $\mu$, because the other parts follow from the previous part of this theorem and [5; Corollary 5.1]. Let $A$ be the $W^{*}$-subalgebra of $M$ generated by $\{p, q, \cdots\}$, then we have

$$
C=A^{\prime} \cap M
$$

where $A^{\prime}$ is the commutor of $A$. Since the centre $M^{\prime} \cap M$ of $M$ is contained in $C$, by Dixmier [1; Proposition 8] (v) holds for any regular gage of $M$.

3. Let $A$ be an abelian $W^{*}$-subalgebra of a semi-finite $W^{*}$-algebra $M$ acting on a separable Hilbert space. Then, by the well known theorem of J. von Neumann [4], there exists a sequence $p, q, \cdots$ of projections in $A$ which generates the algebra $A$, i. e. $\{p, q \cdots\}^{\prime \prime}=A$. Clearly by the definition of the $C\left(=C_{p} \cap C_{q} \cap \cdots\right)$, we have (3) and $C$ is uniquely determined only by $A$ in the equation (3). If $M$ is a semi-finite factor, then the regular gage $\mu$ is essentially unique and the algebre $M_{\mu}$ coincides with the algebra (denoted by $M_{0}$ ) consisting of all the elements of finite rank in the sense of von Neumann [3] which is independent of the regular gage $\mu$. Thus the von Neumann's proposition follows immediately from Theorem 1 :

THEOREM 2 (von Neumann). If $M$ is a semi-finite factor acting on a separable Hilbert space $H$, and if $A$ is an abelian $W^{*}$-algebra generated by its sequence of projections $p, q, \cdots$, then the operation $x \rightarrow x^{|p| q \cdot \cdots}$ defined on the algebra $M_{0}$ (consisting of $x \in M$ with finite rank) depends only on $x$ and $A$, and is independent of the choice or the order of $p, q, \cdots$.

In this theorem, if $M$ is a semi-finite $W^{*}$-algebra with regular gage $\mu$ and 
acting on $H$, then the same fact holds for $M_{\mu}$ without that the operation $x \rightarrow x^{|p| q \mid \cdots}$ depends also on the gage $\mu$, because the algebra $M_{\mu}$ depends on $\mu$. In general, we shall call the operation $x \rightarrow x^{|p| q \cdots}$ to be von Neumann's operation defined by $A .{ }^{1}$ )

4. An abelian $W^{*}$-subalgebra $A$ of a $W^{*}$-algebra $M$ is called to be maximally abelian in $M$ if

$$
A=A^{\prime} \cap M,
$$

or each element of $C$ of (3) is contained in $A$. If $A, M, p, q, \cdots$ are as the beginning in $\S 3$, and if $A$ and $\mu$ are maximally abelian in $M$ and regular gage of $M$, respectvely, then by Theorem 1 the conditional expectation of $\mu$ integrable $x \in M$ conditioned by $A$ coincides with the von Neumann's operation $x \rightarrow x^{|p| q \mid \cdots}$ defined by $A$. Conversely, if the conditional expectation $x \rightarrow$ $x^{e}$ of the $x$ conditioned by $A$ coincides with the operation $x \rightarrow x^{|p| q \mid \cdots,}$, then Theorem 1 implies that $A$ satisfies (4), i.e. $A$ is maximally abelian in $M$. Indeed, putting $C=A^{\prime} \cap M$ and denoting $x \rightarrow x^{\varepsilon}$ the conditional expectation conditioned by $C$, then by Theorem 1 we have $x^{\varepsilon}=x^{\varepsilon}$ for every $x \in M$. Since $A$ is the direct sum of $M^{e}=\left\{x^{e} ; x \in M\right\}$ and $\left\{\lambda\left(I-I^{e}\right) ; \lambda\right.$ being complex numbers\}, cf. [5; Theorem 1], $A=C$. Thus we have the following:

Theorem 3. Let $M$ be a semi-finite $W^{*}$-algebra with a regular gage $\mu$ and acting on a separable Hilbert space. Then an abelian $W^{*}$-subalgebra $A$ of $M$ is maximally abelian in $M$ if and only if the conditional expectation of $x \in M_{\mu}$ conditioned by $A$ coincides with the von Neumann's operation $x \rightarrow x^{i p \mid q . \cdot .}$ defined by $A$.

\section{REFERENCES}

[1] J. Dixmier, Formes linéaires sur un anneau d'opérateurs. Bull. Soc. Math. France 81 (1953), 6-39.

[2] M. Nakamura and T. Turumaru, Expectations in an operator algebra. Tôhoku Math. Journ. 6 (1954), 182-188.

[3] J. von NeumanN, On rings of operators, III. Ann. of Math. 41 (1940), 94-161.

[4] J. von Nedmans, Über Funktionen von Funktionaloperatoren. Ann. of Math. 32 (1931), 191-226.

[5] H. UMEGAKI, Conditional expectations in an operator algebra, II. Tôhoku Math. Journ. 8 (1956), 86-100.

Osaka University of Liberal-Arts and Education,

Tokyo Institute of TeChNOLOGY.

1) If $M$ is a finite $W^{*}$-algebra (not always a factor) acting on a separable Hilbert sprce $H$, and if $A$ is an abelian $W^{*}$-subalgebra of $M$, then the von Neumann's operation defined by $A$, which coincides with the conditional expectation conditioned by $A^{\prime} \cap M$, depends only on $A$ and defined on every $x \in M$. 